\title{
Invagination Intestinale Aiguë De L'adulte: Aspects Diagnostiqsues, Thérapeutiques Et Étiologiques
}

\author{
James Didier L. \\ Département de Chirurgie et Spécialités Chirurgicales, \\ Hôpital National de Niamey, Niger \\ Chaibou MS.
}

Département d'anesthésie, de Réanimation et des Urgences,

Hôpital National de Niamey, Niger

Saidou A.

Abdoulaye MB.

Alassan MSF.

Département de Chirurgie et Spécialités Chirurgicales,

Hôpital National de Niamey, Niger

\section{Daddy $\boldsymbol{H}$.}

Département d'anesthésie, de Réanimation et des Urgences,

Hôpital National de Niamey, Niger

Adamou $\mathrm{H}$.

Faculté des Sciences de la Santé de l'Université de Zinder, Niger Adakal $O$.

Faculté des Sciences de la Santé de l'Université de Maradi, Niger

Marouf MI.

Maternité Issaka Gazoby (enlever le gras), Niamey

Maternité Issaka Gazoby

Sidibe T.

Service de Radiologie à l'Hôpital National de Niamey, Niger

Sani $R$.

Département de Chirurgie et Spécialités Chirurgicales, Hôpital National de Niamey, Niger

Doi: 10.19044/esj.2017.v13n33p265 URL:http://dx.doi.org/10.19044/esj.2017.v13n33p265

Abstract
Introduction: Acute intussusception is a rare clinical entity in adults
where it accounts for only $1-2 \%$ of intestinal obstructions. The authors
wanted to report cases of acute intussusception in adult patients, their
diagnostic aspects, their management, and their etiologies. Patients and 
Methods: This study is a retrospective study of the medical files of patients of both sexes. They include adults over 15 years of age, operated between January 2010 and December 2014, who were diagnosed with obstruction due to acute intestinal intussusception. Results: Six cases of adult intestinal intussusception were collected. The average age was 26.5 years. The sex ratio was 1 and there were so many men as women. Five out of six patients were unstable on admission. The diagnosis was made preoperative in two cases out of six, 33.3\%. Ultrasound revealed a target sign in two cases. The ileo-ileal form was the most frequent (5 cases out of 6) or $83.3 \%$. Also, there were two cases of intestinal necrosis out of six. Intestinal resection was performed in five cases or $83.33 \%$. The cause of intussusception was found in four cases out of six or $66.7 \%$. Here, a tumor was the cause in half of the cases. Immediate surgical follow-up was uncomplicated in all patients. Conclusion: Acute intestinal intussusception of the adult is a very rare condition. The preoperative diagnosis of acute intussusception of the adult remains delicate. The ileo-ileal form is more frequent than the ileo-colic form in adults. Treatment is always surgical in adults.

Keywords: Acute intestinal intussusception, obstruction, adult, Niamey, Niger

\section{Résumé}

Objectifs: L'invagination intestinale aiguë est une entité clinique rare chez l'adulte où elle ne représente que 1 à $2 \%$ des occlusions intestinales. Les auteurs ont voulu rapporter les cas d'invagination intestinale aigüe de l'adulte, leurs aspects diagnostiques, leurs prises en charge et leurs étiologies. Patients et méthodes : Il s'agissait d'une étude rétrospective portant sur les dossiers de patients des deux sexes, adultes âgés de plus de 15 ans, opérés entre janvier 2010 et décembre 2014 dont le diagnostic d'occlusion sur invagination intestinale aigüe a été posé. Résultats : Six cas d'invagination intestinale aiguë de l'adulte avaient été colligés. L'âge moyen était de 26,5 ans. Le sexe ratio était de 1, il y'avait autant d'hommes que de femmes. Cinq patients sur 6 avaient été reçus eu urgence. Le diagnostic était posé en préopératoire dans deux cas sur six soit 33,33\%. L'échographie avait montré une image en «cible» dans deux cas. La forme iléo-iléale a été la plus fréquente ( 5 cas sur 6 ) soit $83,34 \%$. Il y avait deux cas de nécrose intestinale sur six. La résection intestinale avait été effectuée dans cinq cas, soit $83,34 \%$. La cause d'invagination avait été retrouvée dans quatre cas sur six, soit $66,67 \%$, dont une cause tumorale dans la moitié des cas. Les suites opératoires immédiates ont été simples chez tous les patients. Conclusion: L'invagination intestinale aigüe de l'adulte est une pathologie très rare. Le diagnostic préopératoire de l'invagination intestinale aiguë de l'adulte reste 
délicat. La forme iléo-iléale est plus fréquente que la forme iléo-colique chez l'adulte. Le traitement est toujours chirurgical chez l'adulte.

Mots-clés: Invagination intestinale aiguë, occlusion, adulte, Niamey, Niger

\section{Introduction}

L'invagination intestinale aiguë est définie par le télescopage et la pénétration d'un segment intestinal (anse invaginée) dans le segment d'aval (anse invaginante ou réceptrice) à la suite d'un péristaltisme modifié (Darouichi, 2011). Elle est la première cause d'occlusion chez le nourrisson où elle survient le plus souvent au cours d'une pathologie bénigne. C'est une entité clinique qui est rare chez l'adulte où elle ne représente que 1 à $2 \%$ des occlusions intestinales avec une cause organique dans $70 \%$ à $90 \%$ (Traoré, 2012). Le diagnostic est difficile et le plus souvent fait en peropératoire devant une occlusion (Boubbou, 2009). Chez l'enfant c'est une pathologie bénigne. Par contre chez l'adulte elle est très souvent associée à une tumeur intestinale. Le traitement est toujours chirurgical chez l'adulte et ne laisse souvent pas place à la réduction par hyperpression sous contrôle radiologique (Boubbou, 2009). L'anatomopathologie est nécessaire pour confirmer le diagnostic étiologique. Le pronostic est lié à la durée d'évolution, à l'étendue des lésions, à la nature de la cause (Boubbou, 2009).

\section{Patients et Méthodes}

Il s'agit d'une étude rétrospective descriptive allant du $1^{\mathrm{er}}$ janvier 2010 au 31 décembre 2014 soit 5 ans. Etaient inclus les patients des deux sexes âgés de plus de 15 ans, chez qui le diagnostic d'invagination intestinale aiguë a été posé (ou de découverte peropératoire) et opérés. N'étaient pas inclus Patients âgés de moins de 15 ans opérés pour invagination intestinale aiguë et ceux de plus de 15 ans opérés pour d'autres causes d'occlusions intestinales.

\section{Observations}

\section{Observation $\mathbf{n}^{\circ} \mathbf{1}$}

Patient âgé de 18 ans, sans antécédents pathologiques connus, était hospitalisé pour syndrome subocclusif et masse de la fosse iliaque droite avec notion de douleur abdominale et des vomissements postprandiaux évoluant depuis trois (3) mois et un arrêt des matières et des gaz spontanément résolutif. L'examen clinque avait révélé des douleurs abdominales, un arrêt des matières et des gaz, un ballonnement abdominal et une masse à la fosse iliaque droite sensible, de consistance ferme, mobile par rapport au plan profond. La radiographie de l'abdomen sans préparation avait retrouvé des niveaux hydro-aériques mixtes et une échographie qui 
avait révélé une image en «cible» évoquant une Invagination intestinale aiguë. L'exploration chirurgicale avait révélé une invagination iléo-caecocolique, dont la désinvagination était impossible, il avait été réalisé une hémi-colectomie droite et une anastomose immédiate iléo-colique terminolatérale. La désinvagination de la pièce opératoire après la résection n'avait pas révélé de masse, ni d'adénopathie. L'examen anatomo-pathologique de la pièce opératoire n'avait rien trouvé de pathologique. Les suites opératoires étaient simples.

\section{Observation $n^{\circ} 2$}

Patiente âgée de 21 ans, sans antécédents connus, était admise aux urgences dans un tableau d'occlusion intestinale aigüe avec douleur abdominale diffuse, arrêt des matières et des gaz et des vomissements postprandiaux précoces. L'examen clinique avait révélé un ballonnement abdominal diffus avec présence des selles dans l'ampoule au toucher rectale. Une radiographie de l'abdomen sans préparation avait révélé des niveaux hydro-aériques de types grêliques évoquant une occlusion intestinale aiguë du grêle. L'exploration chirurgicale avait mis en évidence une invagination iléo-iléale sur $10 \mathrm{~cm}$ environ situé à $160 \mathrm{~cm}$ de la jonction iléo-caecale et des nombreuses adénopathies mésentériques, pas de nécrose, pas de masse, une désinvagination fut réalisée, puis un prélèvement de ganglion pour examen anatomo-pathologique. Les résultats de l'examen histologique rapportaient des adénopathies de types inflammatoires banales. Les suites opératoires étaient simples.

\section{Observation $\mathbf{n}^{\circ} 3$}

Patient âgé de 30 ans, sans antécédents particuliers, était admis aux urgences pour douleur abdominale diffuse et vomissement. L'examen clinique révélait un ballonnement abdominal diffus et une sensibilité abdominale à la palpation. La radiographie de l'abdomen sans préparation avait révélée des niveaux hydro-aériques de types grêliques évoquant une occlusion intestinale aiguë du grêle. L'exploration chirurgicale avait révélé une invagination iléo-iléale secondaire à une tumeur intra-luminale du grêle à $90 \mathrm{~cm}$ de la jonction iléo-caecale, pas de nécrose, pas d'adénopathie, comme geste une désinvagination fut faite puis une résection emportant la tumeur avec anastomose immédiate termino-terminale. L'ouverture de la pièce opératoire après résection avait révélé une masse intraluminale. A l'examen anatomo-pathologique il s'agissait d'un adénocarcinome du grêle. Les suites opératoires étaient également simples. 


\section{Observation $n^{\circ} 4$}

Patiente âgée de 35 ans, sans antécédents pathologiques connus, était admise aux urgences pour douleur abdominale, arrêt des matières et des gaz et des vomissements fécaloïdes. L'examen clinique avait révélé un ballonnement abdominal diffus et une ampoule rectale vide au toucher rectale. L'imagerie avait révélé des niveaux hydro-aériques de types grêliques évoquant une occlusion du grêle. L'exploration chirurgicale révélait une invagination iléo-iléale de $60 \mathrm{~cm}$ de la jonction iléo-caecale, il y avait environ $50 \mathrm{~cm}$ de grêle invaginé sur lequel on notait des lésions de nécrose pré-perforatives. Comme geste une désinvagination fut faite puis une résection emportant en monobloc la tumeur, les lésions de nécrose et préperforatives ensuite une anastomose immédiate termino-terminale fut faite. L'ouverture de la pièce opératoire avait révélé une masse intraluminale. A l'examen anatomo-pathologique il s'agissait d'une tumeur carcinoïde du grêle. Les suites opératoires étaient également simples.

\section{Observation $n^{\circ} 5$}

Patiente âgée de 35 ans, sans antécédents pathologiques connus, était admise aux urgences pour douleur abdominale diffuse, arrêt des matières et des gaz et des vomissements postprandiaux précoces. L'examen clinque avait révélé un ballonnement abdominal diffus, une masse sous ombilicale, de consistance ferme, douloureuse, mobile par rapport au plan profond. Une radiographie de l'abdomen sans préparation révélait des niveaux hydroaériques de types grêliques. Une échographie abdominale qui révélait une image en «cible » évoquant une invagination intestinale aiguë. L'exploration chirurgicale avait objectivé une invagination iléo-iléale secondaire à une tumeur du grêle d'environ $4 \mathrm{~cm}$ de diamètre intra-luminale, présence desadénopathies mésentériques, pas de lésions de nécrose. La désinvagination était impossible. Comme geste, il a été réalisé une résection emportant en monobloc le boudin d'invagination, la tumeur ainsi que les adénopathies mésentériques sur environ $7 \mathrm{~cm}$ des marges saines. Puis anastomose immédiate termino-terminale. La désinvagination et l'ouverture de la pièce opératoire avaient mis en évidence une tumeur du grêle. L'examen anatomo-pathologique macroscopique avait mis en évidence une tumeur pédiculée, obstruante avec un aspect en chou-fleur. L'histologique de la pièce avait objectivé une masse compatible avec un polype adénomateux associé à une angiodysplasie sans aucun signe de malignité. Les suites opératoires étaient simples.

\section{Observation $n^{\circ} 6$}

Patient âgé de 20 ans, sans antécédents pathologiques connus, était admis aux urgences pour douleur abdominale, arrêt des matières et des gaz et 
des vomissements postprandiaux précoces, dans un contexte d'asthénie physique. L'examen clinque révélait un ballonnement abdominal diffus, une ampoule rectale vide au toucher rectale. La radiographie de l'abdomen sans préparation révélait des niveaux hydro-aériques de types. L’exploration chirurgicale avait révélé une invagination iléo-iléale avec des lésions de nécrose, pas de masse palpable. La désinvagination était impossible, comme geste il a été réalisé une résection du boudin d'invagination emportant toutes les lésions de nécrose, ensuite anastomose immédiate termino-terminale. A l'ouveture de la piece operatoire il n'y avait pas de masse, ni d'adénopathies. L'examen anatomo-pathologique était normal. Les suites opératoires étaient simples.

\section{Résultats Globaux}

Durant la période d'étude, 6 cas d'invagination intestinale aiguë chez l'adulte avaient été enregistrés dans les services de chirurgie viscérale de 1'Hôpital National de Niamey. Cela sur un total de 790 cas d'occlusions intestinales parmi 10337 cas d'admission de pathologies. La fréquence moyenne était de 1,2 cas/ an. La fréquence des invaginations intestinales aigues par rapport aux occlusions intestinales était de 0,76\%. Par contre cette fréquence par rapport au total des admissions en chirurgie viscérale était de $0,058 \%$. L'âge moyen des patients était de 26,5 ans avec des extrêmes allant de 18 à 35 ans. Il y'avait autant d'hommes que de femmes, soit un sex-ratio de 1. La douleur abdominale et les vomissements représentaient les signes les plus fréquents (100\% des cas) suivi de l'arrêt des matières et des gaz dans 83,34\%. A l'examen physique, Deux(2) patients sur 6 soit 33,33\%, présentaient une masse palpable. La radiographie de l'abdomen sans préparation, pratiquée chez tous les patients (100\%) avait révélé des niveaux hydro-aériques. L'Echographique, Pratiquée dans un tiers de cas, a révélé une image en «cible», évoquant une invagination intestinale. Aucun des patients de cette série n'avait bénéficié d'une exploration par scanner. Le diagnostic était posé en préopératoire dans deux cas sur six soit $33,3 \%$. Cinq patients ont été opérés en urgence $(83,34 \%$ des cas) et un patient (16,66\% des cas) en urgence différé. La voie d'abord a été une laparotomie. L'exploration chirurgicale avait mis en évidence un boudin d'invagination de type iléo-iléale chez 5 patients soit $83,34 \%$ des cas et un boudin d'invagination de type iléo-caeco-colique chez un patient $(16,66 \%)$. il y’avait une nécrose intestinale et des adénopathies mésentériques dans $\operatorname{deux}(2)$ cas chacun soit 33,33\%. Le geste opératoire avait été variable selon les cas. Une résection du grêle avec anastomose grêlo-grêlique terminoterminale a été réalisée dans deux tiers des cas. Parmi ces quatre patients, deux ont bénéficié d'une désinvagination intestinale avant la résection. Une hémicolectomie droite suivie d'un rétablissement immédiat de la continuité a 
été effectuée chez un patient porteur d'une invagination iléo-caeco-colique. Une désinvagination sans résection a été effectuée chez l'autre patient. Les suites opératoires immédiates étaient simples chez tous les patients. Aucun cas de décès post-opératoire n'a été noté dans la série. Les différentes causes des invaginations intestinales étaient une tumeur du grêle dans la moitié des cas soit $50 \%$. Dans un cas $(16,67 \%)$ il s'agissait des adénopathies et dans les deux autres cas $(33,33 \%)$ il n'y avait d'obstacle évident responsable de l'invagination. L'examen anatomopathologique des pièces opératoires avait révélé un polype adénomateux associé à une angiodysplasie sans de signe de malignité, des adénopathies inflammatoires banales, un adénocarcinome du grêle et une tumeur carcinoïde du grêle dans un cas chacun.

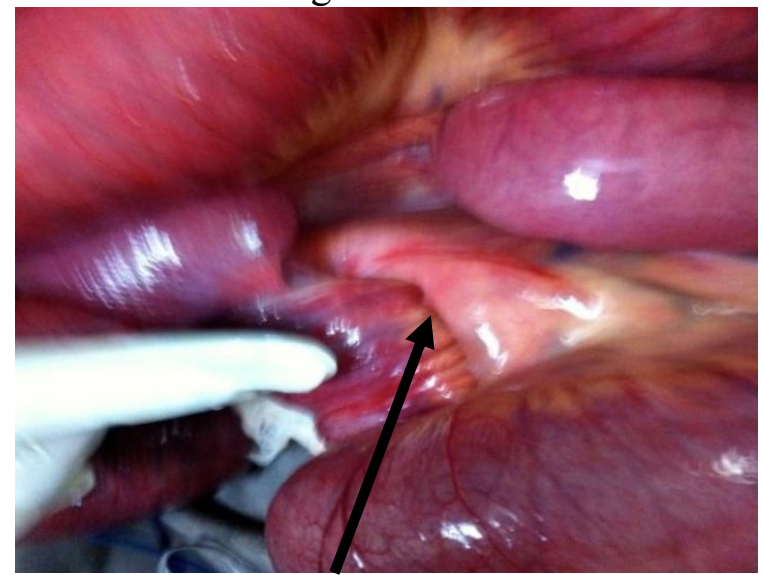

Image 1. invagination ileo-ileale chez un adulte

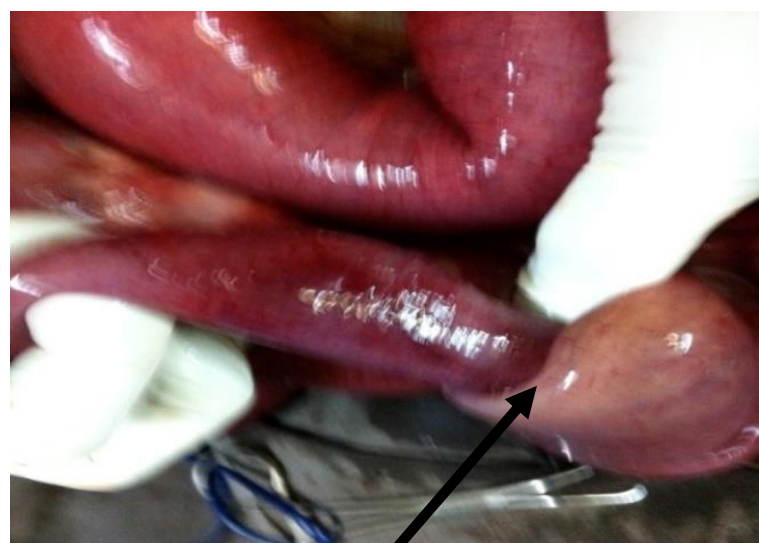

Image 2. Tentative de réduction infructueure de l'invagination ileo-ileale chez un adulte 


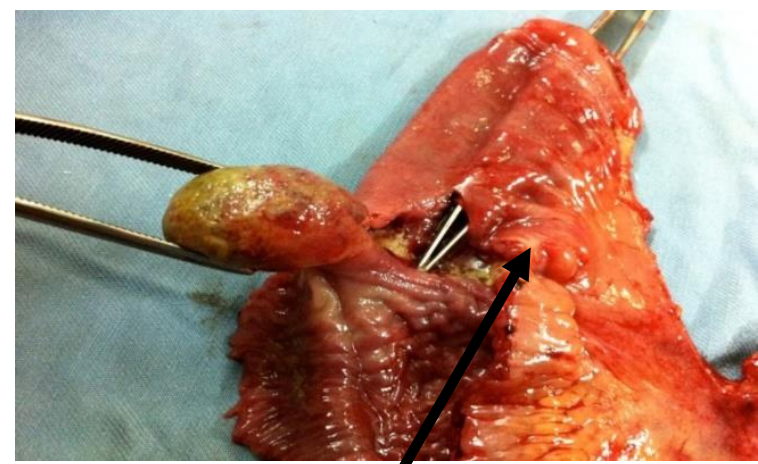

Image 3. Découverte d'une tumeur à l'ouverture de la pièce opératoire

\section{Discussion}

Durant la période de l'étude, 6 cas d'invaginations intestinales aigues de l'adulte en 5 ans ont été enregistrés sur un totale de 790 cas d'occlusions intestinales, soit une fréquence annuelle de 1,2 cas/an et une fréquence de $0,76 \%$ par rapport aux occlusions intestinales. La rareté de l'invagination intestinale aigüe de l'adulte est également rapportée en occident comme au canada (Zubaidi, 2006) et en France (Barussaud, 2006) avec respectivement 22 cas en 18 ans (1,2/an) et 44 cas en 25 ans (1,76/an), mais aussi en Afrique où, D.Traore et al ont rapporté 41 cas en 28 ans soit 1,48/an (Traoré, 2012). L'âge moyen est de 26,5 ans avec des extrêmes allant de 18 à 35 ans. Il s'agit donc de l'adulte jeune comme rapporter par plusieurs auteurs où cet âge se situe entre 33 ans et 44 ans (Traoré, 2002; Traoré, 1999; Ayité, 1993). Le sexe ratio est de 1 soit autant d'hommes que de femmes. M.Boubbou et al ont rapporté des résultats similaires avec aussi un sexe ratio de 1 (Boubbou, 2009). Par contre D.Traore et al ainsi que d'autres auteurs rapportent une prédominance masculine (Traoré, 2012; Eihattabi, 2012). La majorité des patients sont reçus en urgence. Dans la série de D.Traore et al, 93\% des patients étaient admis en urgence (Traoré, 2012). Les patients de notre série présentent des douleurs abdominales, les mêmes résultats ont été rapportés par certains auteurs (Desai, 1999; Lebeau, 2006). Les vomissements ont été notés également chez tous les patients, ces résultats sont plus élevés que ceux rapportés dans d'autres séries (Dennis, 1997; Jain, 2006; Carneiro, 2004; Delannoy, 1999). L'arrêt des matières et des gaz est présent dans la majorité des cas, certains auteurs ont rapporté ce signe dans $25 \%$ à $63 \%$ des cas (Traoré, 2012; Boubbou, 2009). Au cours de l'examen physique, la palpation abdominale est un élément fondamental de grande valeur diagnostique; une masse abdominale est palpée dans un tiers des cas dans notre série; des auteurs ont rapporté des chiffres compris entre $17,64 \%$ à $58,5 \%$ (Eihattabi, 2012). En raison de la difficulté du diagnostic clinique de l'invagination intestinale aigüe de l'adulte, les explorations radiologiques jouent un rôle primordial dans le diagnostic pré-opératoire de cette 
pathologie. L'abdomen sans préparation est réalisée chez tous les patients, et a révélé des niveaux hydroaériques de types grêliques dans la majorité des cas et des niveaux hydro-aériques mixtes chez un patient, ce résultat se rapproche de celui de Lebeau et al. (Lebeau, 2006). Par contre dans d'autres publications les niveaux hydro-aériques étaient présents dans $50 \%$ à $88 \%$ des cas (Boubbou, 2009; Zubaidi, 2006). L'échographie abdominale réalisée chez un tiers des patients a révélé une image «cible» en faveur d'une invagination intestinale. Cet examen est peu demandé du fait que l'invagination intestinale comme cause de l'occlusion n'a pas été suspecté en première intention. Selon les auteurs l'image échographique en faveur d'une invagination intestinale est retrouvée dans $50 \% 85,8 \%$, voire $92 \%$ des cas (Zubaidi, 2006; Dennis, 1997). Le traitement de l'invagination intestinale de l'adulte est chirurgical en raison de la haute incidence des lésions organiques causales. Le choix de la méthode chirurgicale reste influencé par le site, la taille, la cause et la viabilité de l'intestin invaginé. La laparotomie médiane à cheval sur l'ombilic a été réalisée chez tous les patients. La même voie d'abord a été rapportée par plusieurs auteurs (Lebeau, 2006; Carneiro, 2004). A l'exploration une nécrose intestinale a été observée dans un tiers de cas, Bazira avait rapporté le même constat (Bazira, 1990). Par contre Lebeau et al avait rapporté 50\% de nécrose (Lebeau, 2006). L'invagination iléo-iléale est plus fréquente que la forme iléo-caeco-colique. Plusieurs séries ont rapporté une prédominance de la forme iléo-iléale (Traoré, 2013; Eisen, 1999; Ghaderi, 2010). Une résection intestinale a été réalisée chez 5 patients et une réduction simple chez un patient. Ces résultats se rapprochent de ceux d'autres auteurs qui avaient rapporté un taux de résection intestinale à $95 \%$ des cas contre une réduction simple à $5 \%$ des cas (Traoré, 2012; Lebeau, 2006; Klein, 2000). Eihattabi et al ont rapporté un taux de résection à $100 \%$ (Eihattabi, 2012). Une tumeur est retrouvée dans la moitié des cas, des adénopathies dans un sixième des cas; et une absence d'obstacle dans un tiers des cas. Plusieurs auteurs avaient rapporté une étiologie tumorale dans 41,4\% à 74\% (Traoré, 2013; Lebeau, 2006; Eihattabi, 2012). M. Boubbou et al ont rapporté une étiologie tumorale dans $100 \%$ des cas (Boubbou, 2009) Les suites opératoires immédiates sont simples dans cette série par contre D. Traoré et al ont rapporté un taux de mortalité de 5\% (Traoré, 2012).

\section{Conclusion}

L'invagination intestinale aigüe de l'adulte est une pathologie très rare, dont l'une des principales causes est tumorale mais peut aussi être sans cause objectivée. Le diagnostic préopératoire de l'invagination intestinale aiguë de l'adulte reste délicat, une amélioration des moyens diagnostics dans nos pays en voie de développement est nécessaire. La forme iléo-iléale est 
plus fréquente que la forme iléo-colique chez l'adulte. Le traitement est toujours chirurgical chez l'adulte, la résection était plus fréquente que la désinvagination simple.

\section{References:}

1. Ayite, A.E., Sako, A.S., \& Noma M et al. (1993). Les invaginations intestinales aigues de l'adulte au Niger. Médecine d'Afrique Noire; 40(12):746-753.

2. Barussaud, M., Regenet, N., Briennonx et al. (2006). Clinical spectrum and surgical approach of adult intussusceptions: a multicentric study; Internationale Journal of colorectal diseases.dec;21(8):834-839.

3. Bazira, L., Ndayisaba, G., Armstrong, O., \& minani MKA- Rayouba, R. (1990). L'invagination intestinale aigüe. A propos de 18 cas à Bujumbura. Médecine d'Afrique Noire,37(10):546-549.

4. Boubbou, M., Idrissi, M. \& Chraibi et al. (2009). Invagination intestinale aigueaigüe de l'adulte; feuillets de radiologie;4 9(2).99104.

5. Carneiro, P.M.R., \& Kissusi, M.D et al. (2004). Intussusception seen at muhimbili National hospital, Dar-ES-Salam .East African Medical Journal, september; 81(9) :439-442.

6. Darouichi, M. (2011). Triple invagination intestinale aigüe révélant un syndrome de Peutz-Jeghers, Feuillet de radiologie. (51) :216-221.

7. Delannoy, E. (1999). Invagination intestinale de l'adulte. Rev du praticien, 9, $\mathrm{N}^{\circ} 4,415-419$.

8. Dennis, G., Beges, MD., \& Andreas Sandor et al. (1997). The Diagnosis and management of adult intussusception dennis. The American Journal of Surgery. (173):88-94.

9. Desai, N.,Wayne, M.G ., \& Taub P.J. et al.(1999). Intussusceptions in adults, the mount sinai journal of medicine. 1999 ( 66 ) :336-340 .

10. Eihattabi Khalid et al. (2012). Les invaginations intestinales chez l'adulte à propos de 17 cas. Pan African Medical Journal, (12) :17.

11. Eisen, L.K., Cunningham, J.D., \& Aufese Jr. A.H. (1999). Intussusception in Adults: institutional review. J Am Coll Surg;(188):390-5.

12. F, klein A., \& Hollender, LF. (2000). Les invaginations intestinales de l'adulte (19 obs). Ann. chir. (26). 617-621.

13. Ghaderi, H., Jafarian, A., Aminian, A., \& Daryasari, S.A.M. (2010). Clinical presentations, diagnosis and treatment of adult intussusceptions, a 20 years survey. Int J SURG;( 8):318-2. 
14. Jain, P. \& Heap, S.W et al. (2006). Intussusception of the small bowel discovered incidentally by ct. P .Australian Radiology. (50) :171-174.

15. Lebeau, R., Koffi, E., \& Amani, A et al. (2006). Invaginations intestinales aigues de l'adultes: Analyse d'une séries 20 cas. Annale de chirurgie (131) ; 447-450.

16. Traore, D et al. (2012). Invagination intestinale aigüe chez l'adulte : écueils diagnostiques, morbidité, et mortalité dans un pays en voie de développement : journal de chirurgie viscérale. (149).231-234.

17. Traore, S.S., Bonkoungou, G., \& Kirakoya, B et al. (1999). Les invaginations intestinales de l'adulte. A propos de 26 cas et revue de la littérature, rev. cames-serie A.;1 (.01);74-77.

18. Zubaidi, A., Alsaif, F., \& Silverman, R. (2006). Adults intussusceptions: A retrospective review. disease of colon and rectum;(49):1546-1551. 